\title{
Choline acetyltransferase-containing neurons in the human parietal neocortex
}

\author{
V. Benagiano, ${ }^{*}$ D. Virgintino, ${ }^{\circ}$ P. Flace, ${ }^{\circ}$ F. Girolamo, ${ }^{\circ}$ M. Errede, ${ }^{\circ}$ L. Roncali, ${ }^{\circ}$ G. Ambrosi ${ }^{\circ}$ \\ *Dipartimento di Scienze Biomediche, Facoltà di Medicina e Chirurgia Università di Foggia; \\ 'Dipartimento di Anatomia Umana e Istologia, Facoltà di Medicina e Chirurgia, Università di Bari, Italy
}

(C)2003, European Journal of Histochemistry

A number of immunocytochemical studies have indicated the presence of cholinergic neurons in the cerebral cortex of various species of mammals. Whether such cholinergic neurons in the human cerebral cortex are exclusively of subcortical origin is still debated. In this immunocytochemical study, the existence of cortical cholinergic neurons was investigated on surgical samples of human parietal association neocortex using a highly specific monoclonal antibody against choline acetyltransferase (ChAT), the acetylcholine biosynthesising enzyme. ChAT immunoreactivity was detected in a subpopulation of neurons located in layers II and III. These were small or medium-sized pyramidal neurons which showed cytoplasmic immunoreactivity in the perikarya and processes, often in close association to blood microvessels. This study, providing demonstration of ChAT neurons in the human parietal neocortex, strongly supports the existence of intrinsic cholinergic innervation of the human neocortex. It is likely that these neurons contribute to the cholinergic innervation of the intracortical microvessels.

Key words: human cerebral cortex, cholinergic neurons, microvessels, choline acetyltransferase, immunocytochemistry.

Correspondence: Dr. Vincenzo Benagiano, MD, Dipartimento di Anatomia Umana e Istologia Policlinico, piazza Giulio Cesare, 70124 Bari, Italy. Phone: international +39.080 . 5478318. Fax:international +39.080.5478353. E-mail: benagiano@anatomia.uniba.it

Paper accepted on March 12, 2003

European Journal of Histochemistry 2003; vol 47 issue 3 [Jul-Sept]:253-256
Cholinergic innervation of the cerebral cortex is considered to be of extracortical origin, coming from acetylcholine-containing neurons situated in subcortical nervous centres, such as the medial basal forebrain. Cholinergic nerve fibres projecting to the cortex may contact intracortical microvessels, a finding that has suggested that ascending cholinergic pathways are involved in the neural control of cortical blood flow (Armstrong, 1986; Vaucher and Hamel, 1995). In the last 20 years, a number of studies carried out using immunocytochemistry for acetylcholinesterase (AChE) and choline acetyltransferase (ChAT) have demonstrated intrinsic cholinergic neurons within the cerebral cortex. These neurons have been detected in the cortex of rat (Eckenstein and Baughman, 1984; Levey et al., 1984; Nishimura et al., 1988; Chédotal et al., 1994), fetal monkey (Hendry et al., 1987) and cat (Avendaño et al., 1996). The presence of intrinsic cholinergic neurons in the human cerebral cortex is still debated, but some indications of the existence of these neurons have been obtained by means of in situ hybridization and immunocytochemistry (Kasashima et al., 1998, 1999; Oda and Nakanishi, 2000).

This study was undertaken with the aim of further investigating the presence of intrinsic cholinergic neurons in the human neocortex using immunocytochemistry for the acetylcholine biosynthesising enzyme ChAT, known to be the most satisfactory marker for cholinergic nerve cells and processes.

\section{Materials and Methods}

Fresh samples of superior parietal association neocortex (corresponding to Brodmann's area 7) were obtained from surgical pieces removed from 3 patients (ages 45, 52 and 70) suffering from gliomas deeply localized within the white matter of the parietal lobe. The samples were fixed by immersion for 3 hours at $4^{\circ} \mathrm{C}$ in Bouin's fluid devoid of acetic acid, 


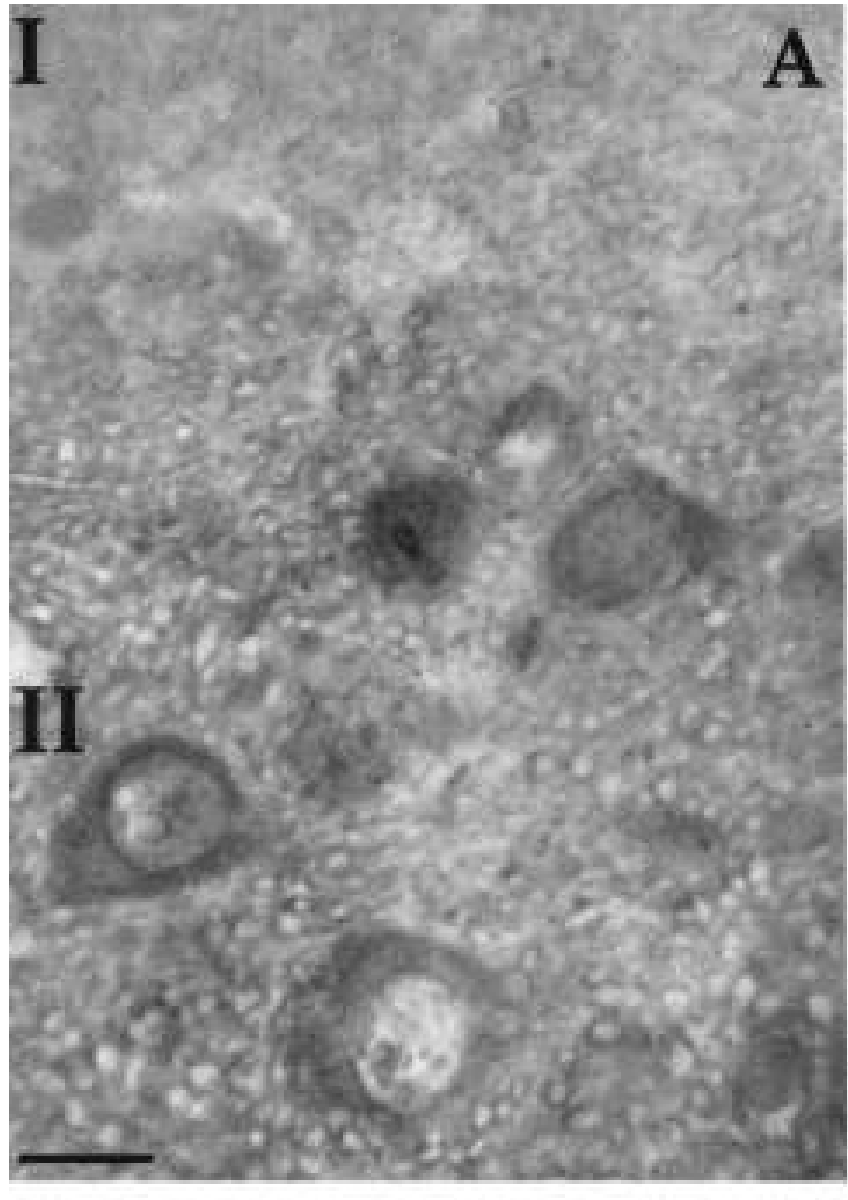

Figure 1. ChAT-positive neurons within layers II and III of the superior parietal association neocortex. (A) Layers I and II: stained pyramidal neurons in layer II. (B) Layer III: one negative and two positive pyramidal neurons; the arrow points the apical dendritic origin of the unstained neuron. (C) Layer III: group of stained pyramidal neurons. Scale bars: A: $30 \mu \mathrm{m}$; B: $20 \mu \mathrm{m}$; C: $15 \mu \mathrm{m}$.

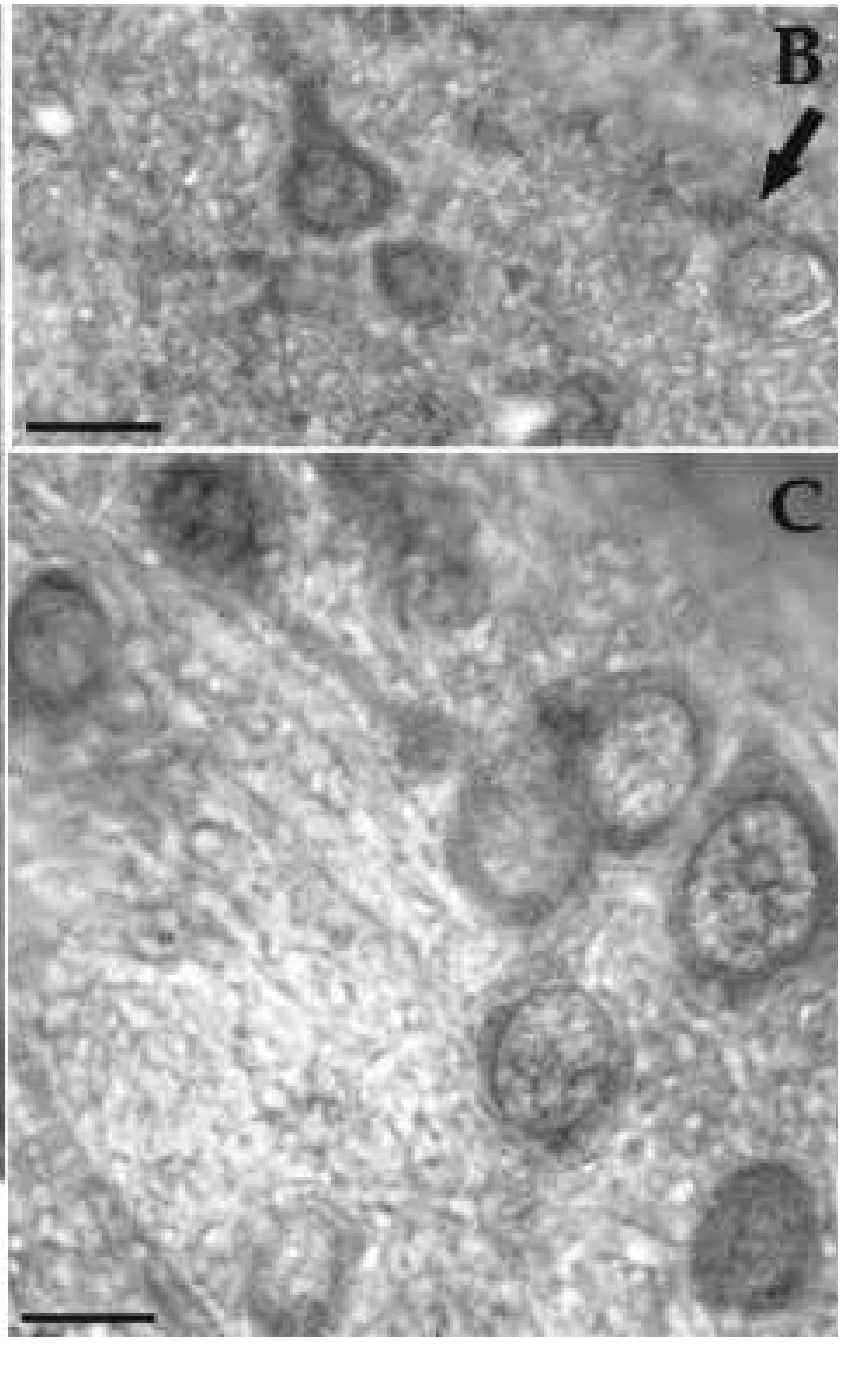

embedded in paraffin and cut into $5 \mu \mathrm{m}$ thick sections. Randomly chosen sections from each sample were stained with toluidine blue and subjected to conventional histopathological analysis. 10 Sections from each sample, evaluated as normal by the histopathologist, were subjected to immunocytochemistry for ChAT using a monoclonal anti-ChAT antibody (Boehringer, Mannheim, Germany) at a final concentration of $20 \mathrm{\mu g} / \mathrm{mL}$ in blocking buffer ( $5 \%$ foetal calf serum, $0.1 \%$ Triton X-100 in Tris-buffered saline, $\mathrm{pH}$ 7.6). The immunocytochemical procedure was performed using the streptavidin-biotin-peroxidase technique ( $L S A B$, Dako, USA) with chromogen 3-amino-9-ethyl-carbazole (AEC, Vector, USA). The sections were counterstained with haematoxylin. Negative control sections were prepared by substituting the anti-ChAT antibody either with the blocking buffer or with an inappropriate monoclonal antibody of identical subclass at the same working dilution. No specific immunostaining was observed in the control sections.

\section{Results}

In the neocortex, ChAT immunoreactivity was detectable in the neuropil as a network of positive neuronal processes that showed a certain degree of specificity in their laminar distribution. Stained processes were more numerous in layers I-III (Figure IA, B) than in layers IV-VI, where only small contingents of processes showed ChAT immunoreactivity. Within layers II and III, small and medium-sized pyramidal neurons, isolated or gathered in small groups, were ChAT-positive and showed a finely granular immunoreactivity within their cytoplasm (Figs. 1, 2). Unstained pyramidal 

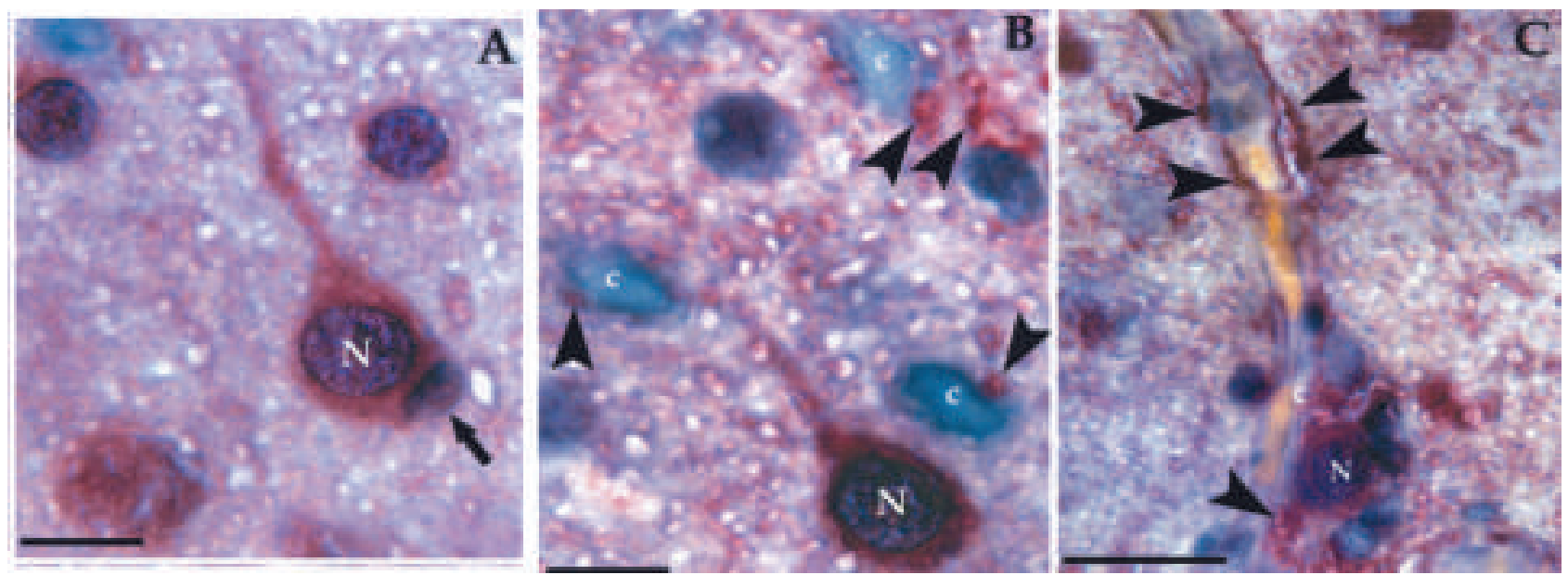

Figure 2. ChAT-positive neurons in layers II and III of the superior parietal association neocortex. (A) Small group of positive neurons in layer III. A medium-sized pyramidal neuron (N) shows immunoreactivity in the perikaryon and apical dendrite; an unstained perisomatic cell (arrow) envelops this neuron. (B) The positive apical process of a pyramidal neuron (N) of layer III is directed towards a cortical capillary (c). The thin walls of the capillaries (c) are tightly surrounded by immunoreactive nerve processes (arrowheads). (C) The perikaryon of a small pyramidal neuron $(\mathrm{N})$ of layer II is in close apposition to a microvessel. Note the positive nerve processes on the contour of the microvessel (arrowheads). Scale bars: $20 \mu \mathrm{m}$.

neurons were also observed in these layers (Figure 1B). The basal surface of the body of positive neurons was often surrounded by unstained small cells (Figure 2A). ChAT immunoreactivity extended into neuronal processes, including the apical dendrites (Figures 1B, 2A, B). Positive nerve processes in close proximity to the wall of microvessels were observed in all layers, and were more numerous in layers II and III (Figure 2C). ChAT-positive neurons closely adjacent to microvessels were also seen (Figure 2C). In layer I and deeper layers IV-VI, no ChAT-positive neurons were revealed.

\section{Discussion}

In this study, the existence of cholinergic neurons has been demonstrated by immunocytochemistry for ChAT in surgical samples of the human parietal neocortex (Brodmann's area 7) using a highly specific monoclonal antibody (Ostermann et al., 1990). ChAT immunoreactivity was observed in small or medium-sized pyramidal neurons distributed in layers II and III. Recently, by means of the in situ hybridization technique, medium-sized pyramidal neurons with ChAT mRNA signal have been revealed in layer III of some areas of the human neocortex, such as the Brodmann's areas 4, 24 and 26 (Kasashima et al., 1998). In a subsequent study on autoptic samples from human cere- bral cortex, using immunocytochemistry for ChAT, the authors identified cholinergic neurons in layers III-V of several cortical areas (Kasashima et al., 1999). Our observations of ChAT-positive and, respectively, ChAT-unlabelled neurons in the layers II and III indicate the existence of a subpopulation of pyramidal neurons, i.e. projection neurons, which express the acetylcholine synthesising enzyme. In the cerebral cortex of other animal species, bipolar neurons, i.e. local circuit neurons, have been shown to exhibit ChAT positivity (Hendry et al., 1987; Chédotal et al., 1994).

The small, unstained cells surrounding ChAT-positive pyramidal neurons closely resembled the perisomatic gliocytes that were identified previously in the cerebral cortex and recognized as oligodendrocytes (D'Amelio et al. 1990; Ambrosi et al. 1995).

The present results suggest that the nerve processes originating from intracortical cholinergic neurons contribute, together with those coming from subcortical cholinergic neurons, to the rich acetylcholine innervation of the cortex and to the formation of the cholinergic nerve plexuses surrounding the cortex microvessels. This finding, taken together with previous observations, would indicate that cortical and subcortical neurons, as well as neurons of parasympathetic ganglia, are all involved in the regulation of the blood flow in the cerebral cortex (Armstrong, 1986; Suzuki and 


\section{Hardebo, 1993; Vaucher and Hamel, 1995; Morales et al., 1995; Benagiano et al., 2000).}

\section{References}

Ambrosi G, Virgintino D, Benagiano V, Maiorano $E$, Bertossi M, Roncali L. Glial cells and blood-brain barrier in the human cerebral cortex. It J Anat Embryol 1995; 100 Suppl 1:177-84.

Armstrong DM. Ultrastructural characterization of choline acetyltransferase-containing neurons in the basal forebrain of rat: evidence for a cholinergic innervation of intracerebral blood vessels. J Comp Neurol 1986;250:81-92.

Avendaño C, Umbriaco D, Dykes RW, Descarries L. Acetylcholine innervation of sensory and motor neocortical areas in adult cat: a choline acetyltransferase immunohistochemical study. J Chem Neuroanat 1996;11:113-30.

Benagiano V, Virgintino D, Rizzi A, Errede M, Bertossi M, Troccoli V, et al. Cholinergic nerve fibres associated to microvessels of the human cerebral cortex: a study based on monoclonal immunocytochemistry for ChAT. Eur J Histochem. 2000;44:165-9.

Chédotal A, Cozzari C, Faure MP, Hartman BK, Hamel E. Distinct choline acetyltransferase (ChAT) and vasoactive intestinal polypeptide (VIP) bipolar neurons project to local blood vessels in the rat cerebral cortex. Brain Res 1994;646:181-93.

D'Amelio F, Eng LF, Gibbs MA. Glutamine synthetase immunoreactivity is present in oligodendroglia of various regions of the central nervous system. Glia 1990;3:335-41.

Eckenstein F, Baughman RW. Two types of cholinergic innervation in cortex, one colocalized with vasoactive intestinal polypeptide. Nature 1984;309:153-5.
Hendry SHC, Jones EG, Killackey HP, Chalupa LM. Choline acetyltransferase-immunoreactive neurons in fetal monkey cerebral cortex. Dev Brain Res 1987;37:313-7.

Kasashima S, Kawashima A, Muroishi Y, Futakuchi H, Nakanishi I, Oda $Y$. Neurons with choline acetyltransferase immunoreactivity and mRNA are present in the human cerebral cortex. Histochem. Cell Biol 1999;111:197-207.

Kasashima S, Muroishi Y, Futakuchi H, Nakanishi I, Oda Y. In situ hybridization study of the distribution of choline acetyltransferase in the human brain. Brain Res 1998;806:8-15.

Levey AI, Wainer BH, Rye DB, Mufson EJ, Mesulam MM. Choline acetyltransferase-immunoreactive neurons intrinsic to rodent cortex and distinction from acetylcholinesterase-positive neurons. Neurosci 1984; 13:341-53.

Morales MA, Holmberg K, Xu ZQ, Cozzari C, Hartman BK, Emson P, et al. Localization of choline acetyltransferase in rat peripheral sympathetic neurons and its coexistence with nitric oxide synthase and neuropeptides. Proc Natl Acad Sci USA 1995;92:11819-23.

Nishimura $Y$, Natori M, Mato M. Choline acetyltransferase immunopositive pyramidal neurons in the rat frontal cortex. Brain Res 198;440:144-8.

Oda $Y$, Nakanishi I. The distribution of cholinergic neurons in the human central nervous system. Histol Histopathol 2000;15:82534.

Ostermann C, Dickmann U, Muley T, Mader M. Large-scale purification of choline acetyltransferase and production of highly specific antisera. Eur. J. Biochem 1990;192:215-8.

Suzuki N, Hardebo JE. The cerebrovascular parasympathetic innervation. Cerebrovasc. Brain Metabol Rev 1993;5:33-46.

Vaucher E, Hamel E. Cholinergic basal forebrain neurons project to cortical microvessels in the rat: electron microscopic study with anterogradely transported Phaseolus vulgaris leucoagglutinin and choline acetyltransferase immunocytochemistry. J Neurosci 1995; 15:7427-41. 